\title{
SEDACIÓN EN CUIDADOS PALIATIVOS: REFLEXIONES ÉTICAS
}

\author{
Josep Porta i Sales MD, PhD. \\ Máster en Bioética
}

Correspondencia: Dr. Josep Porta i Sales, Unidad de Cuidados Paliativos Hospital de la Sta. Creu

Rambla Hospital, 52

08500 Vic (Barcelona)

España 



\title{
SEDACIÓN EN CUIDADOS PALIATIVOS: REFLEXIONES ÉTICAS
}

\author{
Josep Porta i Sales MD, PhD.
}

\section{Resumen}

Se describe el marco en que los cuidados paliativos deben manejar los diferentes problemas que los pacientes y las familias pueden tener al final de la vida. La sedación es una maniobra terapéutica utilizada con cierta frecuencia en cuidados paliativos, que sin embargo presentan el riesgo de conculcar algunos principios éticos. Desde nuestro punto de vista, los principios de beneficencia y autonomía son posiblemente los principios éticos mayormente afectados cuando se considera la sedación. Se discuten algunos de los aspectos para prevenir la conculcación de los principios éticos, asimismo se proporcionan algunas sugerencias para ayudar en la toma de decisiones.

PALABRAS-CLAVE: Sedación, Cuidados Paliativos, Bioética.

\section{Resumo}

Apresentam-se situações de aplicação dos cuidados paliativos em circunstâncias que pacientes e familiares devam enfrentar no final da vida. A sedação é uma conduta terapêutica com frequência utilizada em métodos paliativos e que poderá eventualmente transgredir alguns princípios éticos. Em nosso ponto de vista, a prática da sedação pode afetar especialmente os princípios da beneficência e da autonomia. O presente trabalho propõe medidas que visam evitar eventuais transgressões éticas e oferece sugestões para as tomadas de decisões. 


\begin{abstract}
It is described the frame for applying palliative care to the different problems encountered by patients and their families at the end of life. Sedation is a therapeutic manoeuvre frequently used in palliative medicine, that could eventually infringe some ethical principles. From our standpoint, beneficence and autonomy are probably the most affected principles when sedation is considered. Some measures to prevent such violations to occur are discussed, and suggestions intended to guide in decisionmaking are proposed.
\end{abstract}

KEY-WORDS: Sedation; Palliative Care; Bioethics

\section{Résumé}

On décrit le cadre où les soins palliatifs doivent faire face aux différents problèmes que les patients en fin de vie et leurs familles peuvent avoir. La sédation est une manoeuvre thérapeutique utilisée avec une certaine fréquence en soins palliatifs, mais elle a le risque de nuire certains principes éthiques. Dès notre point de vue, les principes de bienfaisance et d' autonomie sont peut-être les plus afectés lorsqu'on réfléchit à la sédation. On discute sur certains aspects pour prévenir l'oubli des quelques principes éthiques. De la même façon, on propose quelques idées pour aider à la prise de décisions.

MOTS CLÉS: Sédation, Soins Palliatifs, Bioéthique 


\section{Introducción}

Tarde o temprano, el estado de difunto nos será concedido a todos, el de enfermo terminal queda reservado para una creciente mayoría, al menos en los países desarrollados o en vías de desarrollo. Esta afirmación inicial se sustenta en un concepto clínicodescriptivo y en un hecho epidemiológico.

Conceptualmente y desde la perspectiva clínica, hay personas que enferman de procesos morbosos incurables, al menos a la luz de los conocimientos actuales de la medicina. Ello es así independientemente del hecho de disponer de tratamientos que puedan posponer la historia natural de la enfermedad hacia la muerte del paciente. A menudo, en el transcurso del tiempo entre el diagnóstico y el fallecimiento se hacen patentes los efectos deletéreos de la enfermedad sobre el cuerpo, afectando todas las dimensiones de la persona. Este sufrimiento no queda reducido, en forma alguna, al ámbito de la persona sino que afecta de forma directa a la familia y también al grupo de personas que desde una perspectiva profesional intenta luchar contra su enfermedad y sufrimiento. Por lo tanto, el conocimiento, no sólo cierto, sino inminente -a menudo de meses-, de la salida del teatro de la vida, este exitus letalis, constituye la fuente primigenia del sufrimiento y de la realidad témporo-espacial del paciente terminal. Las personas que mueren víctimas de los accidentes de tráfico, de procesos patológicos agudos o de la violencia de otros, difícilmente pueden considerarse como enfermos terminales, ya que carecen de este marco témporo-espacial donde se desarrolla la enfermedad y se prevé la muerte. Estas personas no esperan una salida de escena inminente, su salida es instantánea, fulgurante, podríamos considerar que han sido literalmente arrebatados de la vida, lo cual no es menos traumático para los que se quedan, antes al contrario, posiblemente lo es más. Pero esta es otra cuestión, motivo de otras reflexiones.
Epidemiológicamente, en el primer mundo y por razones ampliamente conocidas, como el aumento de la esperanza de vida, mejor control de las complicaciones de las enfermedades crónicas y un estilo de vida altamente competitivo y estresante, se ha llegado a que las causas principales de muerte sean los accidentes de tránsito (paradigma de la muerte inesperada), el cáncer (paradigma de la muerte esperada) y las enfermedades cardio-vasculares, las cuales pueden adoptar, en general, ambos patrones de muerte. Si consideramos sólo el cáncer, en la actualidad, es causa de entre el 20 y el $25 \%$ de los fallecimientos (según el lugar) lo cual supone un incremento entre 10 y 15 puntos porcentuales respecto a la mortalidad por cáncer de principios del siglo XX.

El desarrollo científico general de los últimos 50 años y en particular de la medicina, junto con una situación social globalmente más justa, han contribuido a que vivamos posiblemente la mejor época de la historia de la humanidad (al menos para los nacidos en el primer mundo). Esta magnificencia y esplendor ha producido un cierto deslumbramiento ante los aspectos íntimos de la vida y la muerte. La indigencia humana se ha maquillado con episeguridades. Aseguramos el hogar, el coche, el Estado asegura un poder adquisitivo mínimo, el acceso universal a los recursos sanitarios, pero también hacemos los mal llamados seguros de vida. En resumidas cuentas, un gran avance con innegables beneficios, pero bajo toda esta cosmética que hace la vida más bonita y agradable, subsiste la vulnerabilidad humana, la enfermedad, el sufrimiento y la muerte.

Ante estas episeguridades, la pseudoomnipotencia médica y la fragilidad humana, se ha establecido una dialéctica que permitió hace unos veinte años reconocer que hay y que continúan habiendo enfermos terminales, los cuales tienen necesidades que es preciso atender. A este redescubrimiento se le ha bautizado como cuidados paliativos. 
Los cuidados paliativos no tienen otra finalidad que proporcionar la mejor calidad de vida a los pacientes en situación terminal hasta que llegue la muerte, afrontándola desde la visión del hombre multidimensional e intentando cubrir todos aquellos aspectos somáticos, psíquicos y transcendentes de la persona. Pero como cualquier avance, también ha generado sus propios dilemas.

El uso de la sedación como estrategia terapéutica proporciona elementos para la reflexión ética, debido a que puede conculcar algunos principios. Este trabajo tiene la pretensión de reflexionar sobre tal hecho, partiendo de la descripción de aquellos conocimientos médicos disponibles en la actualidad, el análisis de la toma de decisiones en cuidados paliativos y observar cómo pueden verse afectados los principios éticos, especialmente el de autonomía y beneficencia.

\section{El trasfondo clínico}

La palabra sedación procede del latín sedare que significa calmar, aliviar. El Diccionari Enciclopèdic de Medicina (1) define sedación como "la acción producida por los sedantes", y a estos como "sustancias que disminuyen la sensación de dolor, o más exactamente, la excitación del sistema nervioso central. La acción sedante de muchos medicamentos está relacionada con sus cualidades analgésicas, tranquilizantes e hipnóticas, y el tipo de acción que se alcanza depende de la dosis suministrada".

En cuidados paliativos, la sedación se ha definido como "la administración de fármacos psicoactivos, generalmente neurolépticos o benzodiazepinas, con la intención de controlar algunos síntomas físicos, psicológicos o producir la pérdida de la conciencia en ciertas situaciones dramáticas, así como también el dormir al paciente, sin su conocimiento y/o consentimiento" (2).

La sedación frecuentemente y a nivel popular tiene la connotación de acción defi- nitiva, es decir, de una acción que producirá la pérdida de la conciencia del paciente y además de forma irreversible, este es el caso de una sedación profunda y continua. La realidad clínica es diferente, la sedación admite matices, puede ser una sedación profunda o superficial, continuada o intermitente.

Los datos de que se dispone sólo hacen referencia a una sedación profunda y continuada que, de acuerdo con Barbero y Camell (3), el mayor grado de discusión ética se establece con este tipo de sedación, debido posiblemente a que la muerte puede verse acelerada en un paciente tan frágil.

Esta sedación profunda y continuada se definió como "sedación terminal", la cual hemos definido como: "La administración deliberada de fármacos para producir una disminución suficientemente profunda $\mathrm{y}$ previsiblemente irreversible de la conciencia en un paciente cuya muerte se prevé próxima, con la intención de aliviar un sufrimiento físico y/o psicológico inalcanzable con otras medidas y con el consentimiento explícito, implícito o delegado del paciente" (4). Esta definición incorpora algunos puntos clave que es necesario considerar a la hora de la toma de decisiones sobre la sedación, tales como: la voluntariedad (maniobra deliberada) de la acción de sedar por parte del equipo, el consentimiento del paciente y el no disponer de otras posibilidades terapéuticas (alivio inalcanzable, síntoma refractario).

La frecuencia en que la sedación ha estado indicada como estrategia terapéutica varía de unos autores a otros, oscilando entre el $16 \%$ y el $52 \%$ (ver tabla I), pero considerando globalmente las series publicadas se puede promediar en un $20 \%$ de los pacientes.

Las causas que más frecuentemente llevan a los equipos a la sedación del paciente son la disnea, el dolor y el delirio (ver tabla II). La mirada fría de las cifras indica la gran variabilidad según la muestra estudiada y posiblemente se puedan relacionar fácilmente con las posibilidades y limitaciones de 
cada equipo en la atención de los pacientes y familias. En cualquier caso, sí se podría decir que aquellos síntomas que son capaces de producir un distrés, un sufrimiento más evidente son los que llevan al equipo a utilizar la sedación. Naturalmente, se podría leer al revés: la sedación está indicada en aquellas situaciones de sufrimiento evidente e incontrolable, lo que se ha definido como síntoma refractario (5).

Existe una cuestión central y básica, que es la "refractariedad" del síntoma, es decir: ¿es realmente inaliviable el síntoma? Tal como lo define Cherny et al, "el término refractario puede aplicarse a un síntoma cuando éste no puede ser adecuadamente controlado a pesar de los intensos esfuerzos para hallar un tratamiento tolerable que no comprometa la conciencia". En este sentido, un diagnóstico diferencial acertado y el uso de todas las medidas terapéuticas disponibles constituyen los elementos básicos de buena praxis sin la cual no hay discusión ética posible. Un caso paradigmático es el delirio en la fase terminal de una enfermedad, que en la literatura se ha denominado de diversas maneras: agitación o inquietud terminal o cuadro confusional hiperactivo. El paciente que sufre un cuadro delirante, que por definición tiene un estado cognitivo alterado, ofrece una imagen de malestar y sufrimiento, puede estar agresivo con incontinencia verbal, no reconoce y responde a los estímulos externos de forma incongruente y alterada. La visión del mundo y la realidad está nublada. Otros pacientes pueden ofrecer una imagen de intranquilidad, con una afectación cognitiva ligera o inexistente, sencillamente porque presentan movimientos musculares incontrolados e involuntarios que son interpretados por la familia como sufrimientos debido a su "comprensible" estado de ansiedad. Lo anterior es posible pero generalmente estaremos asistiendo a un cuadro de mioclonias multifocales. Es bueno saber que aún en estas circunstancias, aunque no siempre, los pacientes son susceptibles de tratamiento" (6).
En definitiva, en cuidados paliativos, los autores que han publicado sus series reconocen que la sedación es utilizada en aproximadamente 1 de cada 5 pacientes en los últimos días de vida, pero no hay información en la literatura sobre el proceso que lleva a tomar esta decisión. Tampoco se informa de la frecuencia de uso de la sedación intermitente, de si había o no consentimiento del paciente o de la familia y aunque la intención benevolente es posible, esta no queda especificada en ningún trabajo.

\section{Reflexión ética}

Desde el punto de vista ético, Fondras (2) recoge las actitudes de médicos franceses responsables de unidades de cuidados paliativos respecto al uso de la sedación. Este autor recopiló la opinión de 30 médicos, la cual posiciona en tres grupos. Un $40 \%$ de los médicos era más próximo a una ética moral o deontológica, un $27,7 \%$ adoptaba una posición más utilitarista y un $33 \%$ mantenía un pensamiento "intermedio". En el grupo utilitarista se observaba una mayor indicación de la sedación que obedecía mayoritariamente a una petición del paciente y con escasa discusión con la familia. En este grupo, la sedación no se considera comparable con la eutanasia o con el uso del cóctel lítico. Por el contrario, un $27 \%$ de los facultativos de los grupos moralista e intermedio pensaba que había una relación entre la sedación y la eutanasia.

Más allá del trabajo descriptivo de Fondras, el cual no hace otra cosa que ilustrar la disparidad de posiciones ante la sedación, es importante reflexionar sobre el uso de una maniobra terapéutica determinada que no tiene, en sí misma, otra consideración ética que la voluntad, la intencionalidad con que es aplicada.

En otras disciplinas como la anestesiología o los cuidados intensivos, el no uso de la sedación sería maleficente. Quién puede pensar que un paciente que ha de sufrir una intervención o una intubación, no esté sedado ya 
sea profunda o superficialmente, según sea el procedimiento a seguir, o el caso del paciente psiquiátrico con un estado de excitación y angustia que puede dañar a alguien o a sí mismo. Seguramente en las situaciones anteriores la razón nos indica que no utilizar los medios farmacológicos disponibles sería conculcar el principio de beneficencia.

¿Qué ocurre en cuidados paliativos? ¿Qué hace que todo se vea diferente? Cuando el paciente puede sufrir los mismos problemas, una disnea o un estado de angustia extrema que no responde al tratamiento habitual. Una posible respuesta recae en dos puntos básicos: la intencionalidad y el resultado.

La intencionalidad, en los casos de los pacientes quirúrgicos, en estado crítico o psiquiátrico, en la práctica habitual queda bien definida a priori, evitar el mal. En estos casos, el sufrimiento del paciente. Más allá de cualquier ejercicio de la autonomía del paciente, ¿quién querría ser intervenido sin anestesia, a lo vivo? ¿Consentiría un cirujano llevar un paciente a un desbridamiento de una gangrena o a una laparotomía sin asegurar la confortabilidad del paciente sometiéndolo a una sedación más o menos profunda, aparte de otras consideraciones anestésicas? Seguro que no. ¿Qué familia consentiría en que un enfermo no estuviese sedado antes de afrontar los procedimientos mencionados? Ninguna.

En cuidados paliativos la duda recae en que la intencionalidad sea, precisamente, no maleficente. Que aquello buscado no sea el bien del paciente. Por tanto, una primera cuestión ineludible recae en responder a quién se beneficia cuando se plantea la sedación. ¿Es una huida hacia adelante del equipo? ¿Es la petición de un paciente o de una familia que no ha tenido la oportunidad de contar con suficiente apoyo ante la pérdida? Por tanto, una primera dimensión que hay que explorar es si la omisión de la sedación es maleficente, es decir, que la intención está en evitar el mal y, la segunda, re- cae sobre si el beneficiario será el paciente. En este punto hay un área de conflicto, a mi modo de ver, ya que uno de los principios de los cuidados paliativos dice que la unidad de tratamiento es el paciente y la familia (7). Este enunciado da igual prioridad a la familia y al paciente, de acuerdo con Randall y Downie (8).

El equipo siempre ha de tener en la mente el beneficio del paciente e intentar que los intereses legítimos de la familia sean escuchados y atendidos. Esto incluye que los equipos han de esforzarse en recordar que un punto esencial de los cuidados paliativos es mantener y preservar la dignidad y autonomía del paciente, es decir, que se ha de dar al paciente la información necesaria para que pueda tomar sus propias decisiones. No hemos de ser deshonestos con los pacientes con tal de facilitar a los familiares una decisión difícil. En el caso de que el paciente no pudiese expresar su voluntad, lo cual no es infrecuente, el equipo ha de velar por el beneficio del enfermo incorporando en los elementos de decisión aquellas aportaciones que desde la familia se puedan hacer sobre los valores y deseos del paciente. También es frecuente que la familia no se encuentre en condiciones emocionales o de conocimiento para tomar una decisión importante. En este caso sería claramente maleficente hacia la familia forzarla a tomar una decisión para la cual su grado de autonomía se halla tan limitado. Es responsabilidad del equipo tomar esta determinación y no pasarla a una familia atenazada por el dolor y la incertidumbre.

Respecto al resultado de la sedación, en el contexto del paciente terminal, es altamente probable que sea la muerte. Este resultado, por ser altamente previsible, es un punto de conflicto, ya que a menudo se piensa que no hay mayor mal que la muerte y es preciso evitarla. Siguiendo los ejemplos del paciente quirúrgico, crítico o psiquiátrico, pero especialmente el del paciente crítico, la muerte no es un futuro improbable, más bien es 
una expectativa factible que un paciente grave, sedado y en una unidad de cuidados intensivos pueda fallecer. En este caso la sedación no se plantea como algo relacionado con la muerte del paciente, a pesar de su estado crítico y que la sedación y la intubación también tienen sus efectos secundarios.

Si bien en el caso del paciente crítico la muerte es una posibilidad no lejana considerando su situación clínica, en el paciente terminal la muerte es una certeza, porque su enfermedad también le ha llevado a una situación "crítica".

No quisiera hacer juego de palabras, pero sí analizar un aspecto importante sobre la responsabilidad de los resultados. Hay acciones u omisiones sobre los cuales podemos ser moralmente responsables respecto a los resultados, sería el caso de administrar un veneno a una persona sana con el fin de producirle la muerte. De la acción de dar el veneno se deriva inevitablemente y causalmente la muerte de la persona. En el caso del paciente terminal, la muerte no depende de nuestras acciones u omisiones, la muerte llegará inexorablemente. Los múltiples factores involucrados en la muerte de los pacientes terminales son difíciles de valorar, hay excesivos parámetros a tener en cuenta para que se pueda declarar que una acción u omisión ha sido la causa de la muerte. En el supuesto que una acción como la sedación, bien indicada, se señale como causa de la muerte, sería falso, porque la muerte hubiese llegado igual y además con mayor sufrimiento. Estaríamos hablando del doble efecto o de una valoración riesgo/beneficio.

Donde somos moralmente responsables no es en el resultado (la muerte) sino en el proceso que nos ha llevado a tomar la decisión (la sedación) (6). Es en este proceso de toma de decisiones que el tener en cuenta todos los elementos en juego hará que una decisión sea éticamente buena o mala. Los elementos para una buena toma de decisio- nes son tanto a nivel del saber científico (historia natural de la enfermedad u opciones terapéuticas, disponibilidad de recursos), como a nivel social (valores, creencias, entorno, entre otros). La integración de todo es lo que ayudará a que la decisión final de acción u omisión sea éticamente consistente.

\section{Conclusiones}

En definitiva, la sedación hay que considerarla una maniobra más dentro del repertorio de los cuidados paliativos, pero atendiendo su impacto sobre el paciente $y$, ante el riesgo de conculcar diversos principios éticos, su utilización ha de ser claramente razonada, discutida y plasmada. Existe el riesgo de ser maleficente, no ser beneficente con el paciente y desatender su autonomía. Estos serían los valores en juego que es preciso respetar, porque de otra manera la sedación es una maniobra éticamente inaceptable.

Nos atreveríamos a sugerir algunos puntos guía que pueden ser de ayuda a la hora de analizar éticamente la sedación:

- Establecer un diagnóstico correcto.

- Sopesar los riesgos y los beneficios (maleficencia/beneficencia).

- Asegurarse que la sedación es la única maniobra posible.

- Obrar siempre en beneficio del paciente, basándonos en el conocimiento (previo o actual) de sus valores y objetivos (autonomía).

En el enfermo no autónomo, recoger la información que nos puedan dar sobre los valores y objetivos del paciente, pero no sobrecargar ni forzar a la familia a una toma de decisiones.

- Discutir con el equipo la toma de decisiones y dejarlo escrito en la historia clínica.

- La sedación es un procedimiento que hay que tener en cuenta dentro del armamentarum, pero no se ha de establecer como rutina. 
Tabla I: Frecuencia en que se indicó la sedación:

\begin{tabular}{|l|c|c|}
\hline Autores & Año & $\%$ \\
\hline Ventafridda et al. (9) & 1990 & $\mathbf{5 2}$ \\
\hline Fainsinger et al. (10) & 1991 & $\mathbf{1 6}$ \\
\hline Stone et al. (11) & 1997 & $\mathbf{2 6}$ \\
\hline Ojeda et al. (12) & 1997 & $\mathbf{1 9}$ \\
\hline Porta et al.(13) & 1999 & $\mathbf{2 3}$ \\
\hline
\end{tabular}

Tabla II: Causas por las que se indicó la sedación (en porcentajes)

\begin{tabular}{|l|c|c|c|c|}
\hline Síntoma/autores & Ojeda et al (12) & Stone et al (11) & Ventafridda et al. (9) & Porta et al (13) \\
\hline Disnea & 74 & 20 & 41 & 23 \\
\hline Delirio & 37 & 60 & 14 & 10 \\
\hline Hemorragia & 8 & - & - & 9 \\
\hline Dolor & 6 & 20 & 39 & 23 \\
\hline Nausea/vómitos & 3 & - & - & 6 \\
\hline Angustia & - & 26 & - & 32 \\
\hline
\end{tabular}




\section{Referencias}

1. Oriol Cassasas (ed.). Diccionari Enciclopèdic de Medicina. Acadèmia de Ciències Mèdiques de Catalunya i de Balears. Barcelona: Enciclopèdia Catalana; 1990: 1370.

2. Fondras JC. Sedation and ethical contradiction. European Journal of Palliative Care 1996; 3: 17-20.

3. Barbero J, Camell H. Sedación y paciente terminal: la conciencia perdida. Medicina Paliativa 1997; 4: 170-178.

4. Porta J, Guinovart C, Ylla-Catalá E. Definición y opiniones acerca de la sedación terminal: estudio multicéntrico catalano-balear. Medicina Paliativa 1999; 6: 150-156.

5. Cherny NI, Portenoy RK. Sedation in the management of refractory symptoms: guidelines for evaluation and treatment. Journal of Palliative Care 1994; 10 (4): 31-38.

6. Back IN. Terminal restlessnes in patients with advanced malignat disease. Palliative Medicine 1992; 6: 293-298.

7. Gómez Batiste-Alentorn X. Atenció als malasts terminals. En: Arquebisbat de Barcelona. Delegació de Pastoral de la Salut. El
Malalt terminal i els professionals de la salut. Barcelona: Claret; 1990; 43-55.

8. Randall f, Downie RS. Palliative care ethics: a good companion. Oxford: Oxford University Press; 1996.

9. Ventafridda V, Ripamonti C, De Conno F. Symptom prevalence and control during cancer patients' last days of life. Journal of Palliative Care 1990; 6: 7-11.

10. Fainsinger R, Miller M, Bruera E. Symptom control during the last week of life on a palliative care unit. Journal of Palliatiive Care 1991; 7: 5-11.

11. Stone P, Philips C, Spruyt O, Waight C. A comparison of use of sedatives in a hospital support team and in a hospice. Palliative Medicine 1997; 11: 140-144.

12. Ojeda Martín M, Navarro Marrero MA, Gómez Sancho M. Sedación y enfermo oncológico terminal. Medicina Paliativa 1997; 4: 101-107.

13. Porta J, Guinovart C, Ylla-Català. Estudio multicéntrico sobre la sedación terminal en cuidados paliativos. Medicina Paliativa 1999 En prensa. 